\title{
ANALYSIS OF SIGNAL TRANSITION ACTIVITY IN FIR FILTERS IMPLEMENTED BY PARALLEL MULTIPLIER ACCUMULATOR BASED ON MODIFIED BOOTH ALGORITHM
}

\author{
T.S. Udhaya Suriya ${ }^{1}$ and Dr. P.Rangarajan ${ }^{2}$ \\ ${ }^{1}$ Dept of Biomedical Engineering, Adhiyamaan College Engineering, Hosur. \\ tsu.suriya@gmail.com \\ ${ }^{2}$ Dept of Computer Science and Engineering, \\ RMD College of Engineering and Technology, India \\ rangarajan69@gmail.com
}

\begin{abstract}
The MAC architecture is used in real time digital signal processing and multimedia information processing which requires high throughput. A novel method to estimate the transition activity at the nodes of a multiplier accumulator architecture based on modified booth algorithm implementing finite impulse response filter is proposed in this paper. The input signals are described by a stationary Gaussian process and the transition activity per bit of a signal word is modeled according to the dual bit type (DBT) model. This estimation is based on the mathematical formulation by multiplexing mechanism on the breakpoints of the DBT model.
\end{abstract}

\section{KEYWORDS}

Digital Signal Processing (DSP), MAC, dual-bit type (DBT), multiplexing mechanism, signal transition activity, word level activity

\section{INTRODUCTION}

Multiplier and multiplier-and-accumulator (MAC) [1] are the important elements of the digital signal processors. For example, many filters and channel estimators require FIR or FFT/IFFT computations that MAC units can accelerate efficiently. The Booth multiplier has the highest operational speed and less hardware count.

To increase the speed of MAC, the modified radix-4 Booth's algorithm (MBA) [2] is usually used. The power dissipation in very large scale integration (VLSI) is the major concern. The dynamic power consumed during charging and discharging the load and parasitic capacitances, depends on the number of transitions occurring at the capacitive nodes, estimation of the average Sundarapandian et al. (Eds) : CCSEA, EMSA, DKMP, CLOUD, SEA - 2014 pp. 85-93, 2014. ( ) CS \& IT-CSCP 2014

DOI : $10.5121 /$ csit.2014.4308 
number of bit level signal transitions is a most requirement of the power estimation techniques. Estimation of the data path power consumption at the architectural level based on the dual -bittype (DBT) model has been explained in [3]. In DBT model, the LSB's of a word behaves as random sequences and the MSB's exhibits nonrandom behavior. The bit level activity is explained in [4]. The DBT model and high level signal statistics for the estimation of the bit level transition activity on filters has been proposed in [5]. This paper deals with the accurate calculation of transition activity at the nodes of a MAC which is used in FIR filter, is discussed. The DBT model is followed to calculate required word-level signal statistics at the nodes of the MAC, with known statistics.

\section{MULTiPlier ARCHITECHTURE}

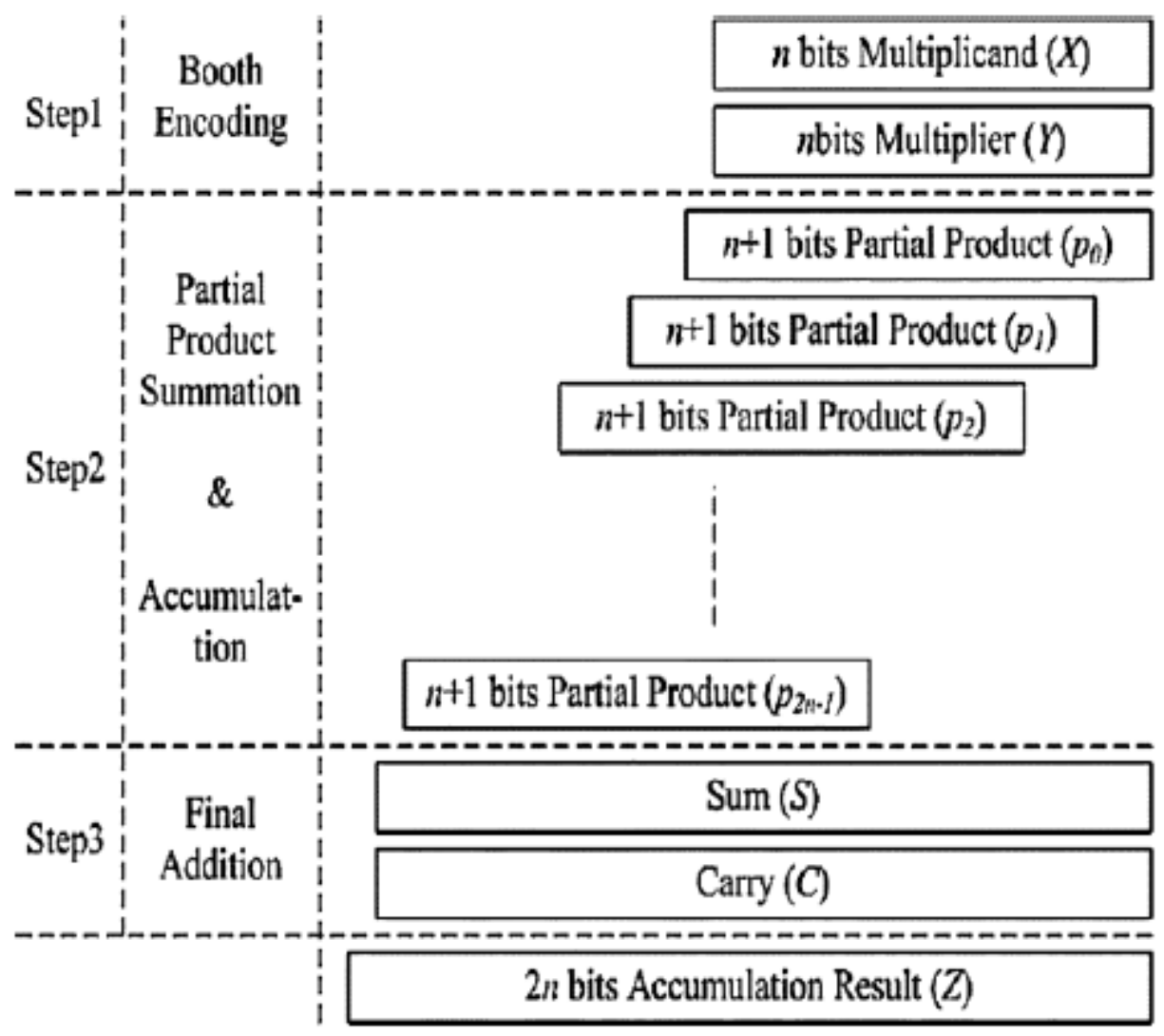

Fig.1. Basic arithmetic operation of multiplication and accumulation

The Multipliers has two parts. The first part is to generate the partial products, and the second one is to collect and add them. The multiplier accumulator composed of three blocks: the booth encoder, partial product summer and accumulator, and final adder. The partial product can be generated by using any one of the multiplication algorithm which uses bit serial, serial-parallel, or full parallel techniques. The booth algorithm or the modified booth algorithm is used for the generation of partial products. The basic arithmetic operation of multiplication and accumulation is shown in fig.1. The signed multiplication based on 2's complement is possible. If N-bit data 
are multiplied the number of partial product also equal to N. the Modified Booth encoding is used to reduce the number of partial products [7].

Muliplicand Multiplier

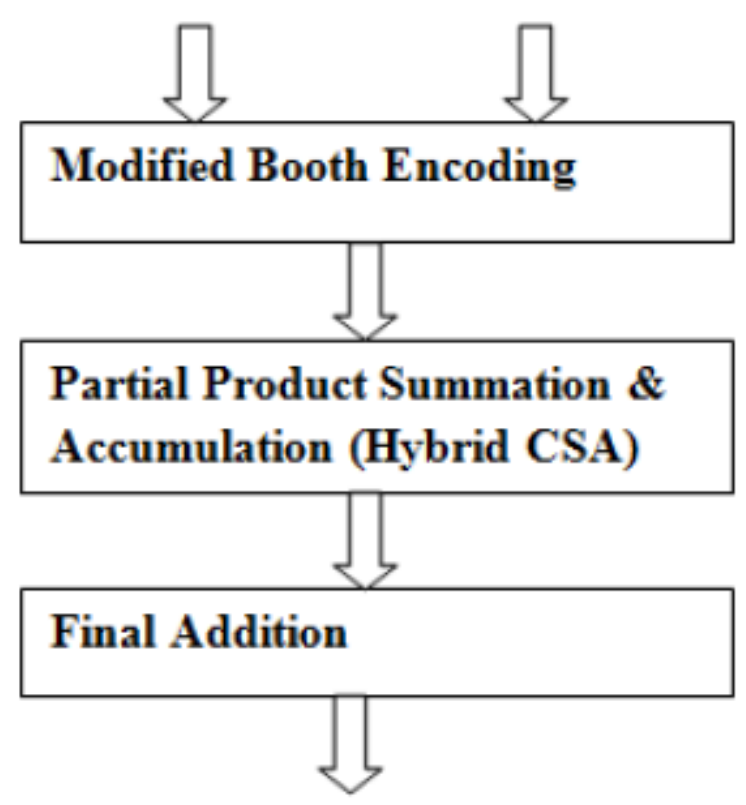

Output

Fig.2. Multiplication process by Modified Booth Encoding.

\section{WORD LEVEL STATISTICS AND TRANSITION ACTIVITY}

The input sequence of a DSP system bex(n) and can be modeled by stationary Gaussian process with mean value $\mu$ variance $\sigma^{2}$, and correlation factor $\rho$. Let $b_{<i>}(n)$, be the ith bit of the binary word representing $\mathrm{x}(\mathrm{n})$. The probability of $\mathrm{b}_{<\mathrm{i}}(\mathrm{n})$ be one or zero and is denoted by $p_{1}^{i}$ and $p_{0}^{i}$ respectively, the conditional probability of $b_{<i>}(n)$ be in state lis given by $b_{<i>}(n-1)$, in state $k$ is denoted by $p_{k l}^{i}$, where $\mathrm{k}, l=0,1$. The transition probability $t p_{k l}^{i}$ is the probability of $\mathrm{b}_{<\mathrm{i}>}(\mathrm{n})=1$ and $\mathrm{b}_{<\mathrm{i}>}(\mathrm{n})=\mathrm{k}$. Therefore $p_{k l}^{i}=p_{k}^{i} p_{k l^{-}}^{i}$ Thus, the transition activity $\mathrm{t}_{\mathrm{i}}$ of $\mathrm{b}_{<\mathrm{i}>}(\mathrm{n})$ is

$$
t_{i}=t p_{01}^{i}+t p_{10}^{i}=p_{0}^{i} p_{01}^{i}+p_{1}^{i} p_{10}^{i}
$$

It is observed that for $p_{0}^{i}=p_{1}^{i}=0.5, p_{01}^{i}=p_{10}^{i}$ and therefore $t_{i}=p_{01}^{i}$

The transition activity is a function of bit level correlation factor $\rho_{<i>}[5]$.

$$
t_{i}=2 p_{1}^{i} p_{0}^{i}\left(1-\rho_{<i>}\right)
$$




\section{BIT LEVEL STATISTICS}

The bit level correlation factor is modeled by DBT model [5],[3]. A signal word is divided into three regions of continuous bits as the LSB, linear and MSB regions. The breakpoints $\mathrm{BP}_{0}$ and $\mathrm{BP}_{1}$ which defines the linear regions given by

$$
\begin{gathered}
B P_{0}=\log _{2}^{\sigma}+\log _{2}^{\left[\sqrt{1-\rho^{2}}+\frac{|\rho|}{8}\right]} \\
B P_{0}=\log _{2}^{3 \sigma}
\end{gathered}
$$

The MSB's are highly correlated signals which have low transition activity while anticorrelated ones possess high activity. The transition activity can be expressed as

$$
t_{i}=\left\{\begin{array}{lr}
2 p_{1}^{i} p_{0}^{i}, & i<B P_{0} \\
\frac{t_{m s b\left(i-B P_{0}\right)-2 p_{1}^{i} p_{0}^{i}\left(i-B P_{1}\right)},}{B P_{1}-B P_{0}}, & B P_{0} \leq i \leq B P_{1} \\
t_{m s b}, & i \geq B P_{1}
\end{array}\right.
$$

Where $t_{\mathrm{msb}}$ is the activity of the MSB. For zero mean Gaussian signals, the word level correlation factor for $t_{\mathrm{msb}}$ is given by

$$
t_{m s b}=(1 / \pi) \cos ^{-1}(\rho)
$$

\section{SIGNAL STATISTICS IN FIR FILTER}

The signal statistics at the output of the $l$ th tap of an FIR filter is the function of the input statistics and the filter coefficients are expressed as

$$
\begin{gathered}
\mu_{f-l}=\mu \sum_{i=0}^{l} c_{i} \\
\sigma_{f-l}^{2}=\sigma^{2} \sum_{i=0}^{l} c_{i}^{2}+2 \sum_{i=0}^{l-1} \sum_{i=0}^{l} \rho(j-i) c_{i} c_{j} \\
\rho_{f-l}=\frac{\sigma^{2}}{\sigma_{f-l}^{2}}\left(\sum_{i=0}^{l-1} c_{i} c_{i+1}+\sum_{i=0}^{l} \sum_{j=0}^{l} c_{i} c_{j} \rho(j-i+1)\right. \\
\left.+\sum_{i=0}^{l-2} \sum_{j=i+2}^{l} c_{i} c_{j} \rho(j-i-1)\right)
\end{gathered}
$$


The structure of FIR filter is shown in fig.3.

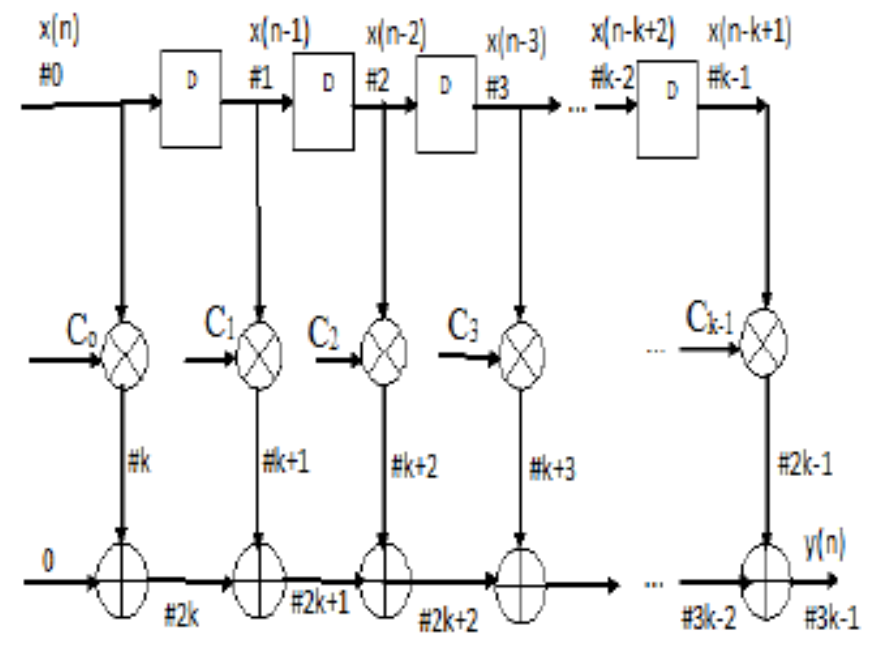

Fig.3.FIR filter structure

\section{MAPPING THE FIR FILTER STRUCTURE ON A MAC BASED ON MODIFIED BOOTH ALGORITHM}

The architecture of FIR filter implemented by MAC using modified Booth algorithm is shown in Fig.4. Registers are inserted at the input and output of the MAC unit to increase the data throughput rate. The sampling period of the input signal at the nodes of the filter structure is a sequence in the time domain at the corresponding nodes of the MAC architecture. During the $\mathrm{n}^{\text {th }}$ input signal sampling period, the sequence $[\mathrm{x}(\mathrm{n}), \mathrm{x}(\mathrm{n}-1), \mathrm{x}(\mathrm{n}-2) \ldots \mathrm{x}(\mathrm{n}-\mathrm{k}+1)]$ appears at the input node \#1 of the MAC. The control signal is given at node \#2, is a increasing integer in the interval $[0, \mathrm{k}-1]$, returning back to zero at every new sample. A mathematical approach to find the statistics of its input sequence is mixing method [8]. The output distribution is a linear combination of the inputs. When all the inputs show identical normal distribution the output distribution remains Gaussian. When all the input sequences have equal mean values and different variances the output distribution is symmetric around the mean value, but not normal. When the input sequences have different mean values, the output distribution is nonsymmetric. 


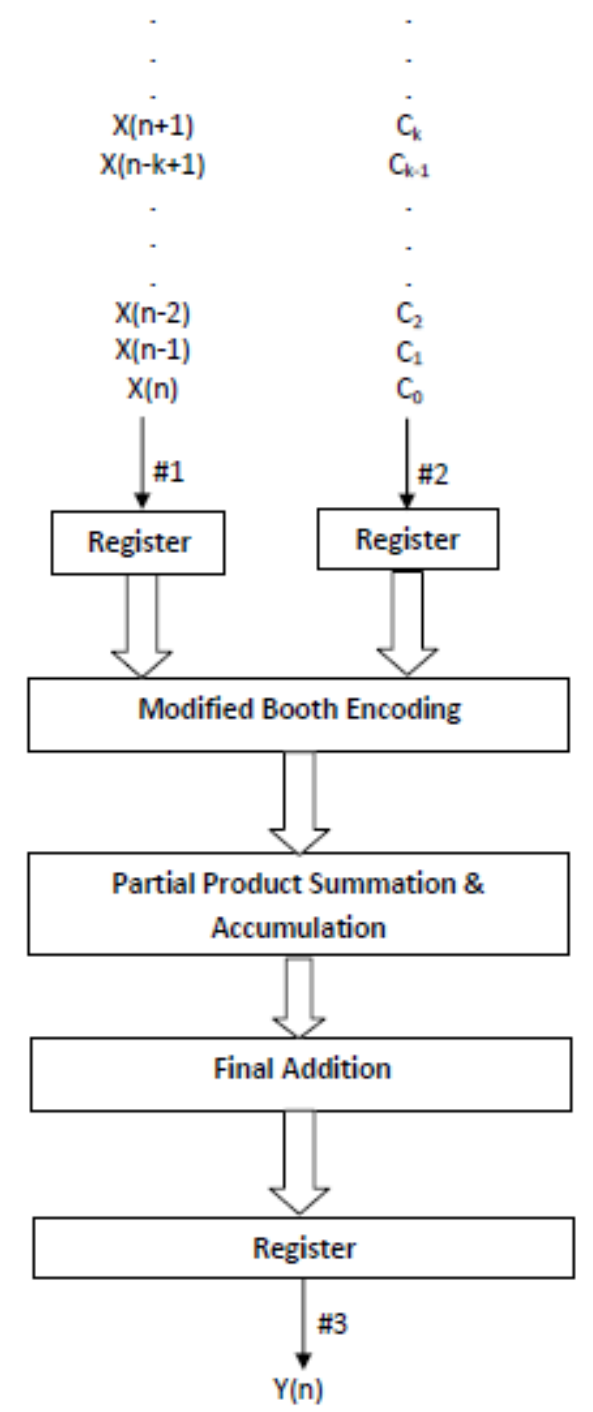

Fig.4.Signal activity at the nodes of MAC unit with Modified Booth Algorithm

\section{SignAL ACTIVITY AT THE NODES OF MAC}

The input signals at the nodes of the MAC unit are the output from the multiplexers. The multiplexing mechanism is used for the signal statistics. The input signal $\mathrm{x}(\mathrm{n})$ is described by a stationary Gaussian process with signal statistics $\mu, \sigma^{2}$ and $\rho$. The statistics of the input signal at node\#1 are given by

$$
\begin{gathered}
\mu_{1}=\mu \\
\sigma_{1}^{2}=\sigma^{2} \\
\rho_{1}=\left(E\left[y_{1}(n) y_{1}(n-1)\right]-\mu_{1}^{2} / \sigma_{1}^{2}\right.
\end{gathered}
$$


Where

$$
\begin{gathered}
E\left[y_{1}(n) y_{1}(n-1)\right] \\
=\frac{1}{k}\left(\sum_{i=2}^{k} E[x(n-i+1) x(n-i+2)]\right. \\
+E[x(n+1) x(n-k+1)])
\end{gathered}
$$

Since the input sequence is assumed to be stationary

$$
\begin{aligned}
& E[x(n-1) x(n)]=\cdots \\
&=E[x(n-k+1) x(n-k+2)] \\
&=\rho \sigma^{2}+\mu^{2} \\
& E[x(n+1) x(n-k+1)]=\rho(k) \sigma^{2}+\mu^{2} \\
& \approx \rho^{k} \sigma^{2}+\mu^{2}
\end{aligned}
$$

The correlation coefficient of $y_{1}(n)$ is $\rho_{1}=$ $\frac{\left((k-1) \rho+\rho^{k}\right)}{k}$

Since $\rho<1$, for higher order filter the correlation coefficient at node\#1 approximates the correlation of the input signal.

The statistics of the sequence of the coefficients at node\#2 is

$$
\begin{gathered}
\mu_{2}=\frac{1}{k} \sum_{i=0}^{k-1} c_{i} \\
\sigma_{2}^{2}=\frac{1}{k} \sum_{i=0}^{k-1} c_{i}^{2}-\mu_{2}^{2} \\
\rho_{2}=\frac{1}{k \sigma_{2}^{2}}\left(\left(\sum_{i=0}^{k-2} c_{i+1} c_{i}\right)+c_{0} c_{k-1}-k \mu_{2}^{2}\right)
\end{gathered}
$$

The statistics of the signal sequence at the output of the multiplier at node\#3 is 


$$
\begin{gathered}
\mu_{3}=\mu_{2} \mu_{1} \\
\sigma_{3}^{2}=\left(\sigma_{2}^{2}+\mu_{2}^{2}\right)\left(\sigma_{1}^{2}+\mu_{1}^{2}\right)-\mu_{3}^{2} \\
\rho_{3}=\left(\left(\rho_{2} \sigma_{2}^{2}+\mu_{2}^{2}\right)\left(\rho_{1} \sigma_{1}^{2}+\mu_{1}^{2}\right)-\mu_{3}^{2}\right) / \sigma_{3}^{2}
\end{gathered}
$$

The sequence at the output nodes \#2k - \#(3k-1) of the filter are given by

$$
\begin{gathered}
\mu_{4}=\frac{\mu}{k} \sum_{j=0}^{k-1} \sum_{i=0}^{j} c_{i} \\
\sigma_{4}^{2}=\frac{1}{k} \sum_{i=0}^{k-1}\left[\sigma_{f-i}^{2}+\mu_{f-i}^{2}\right]-\mu_{4}^{2} \\
\rho_{4}=\frac{1}{k \sigma_{4}^{2}}\left(\sum_{i=0}^{k-2}\left[\sigma_{f-i}^{2}+\mu_{f-i}^{2}\right]\right. \\
+\sum_{i=1}^{k-1} \sum_{j=0}^{i-1} c_{i} c_{j}\left[\rho^{[j-i]} \sigma^{2}+\mu^{2}\right] \\
+c_{0}\left[\sigma^{2} \sum_{i=0}^{k-1} c_{i} \rho^{i+1}+\mu^{2} \sum_{i=0}^{k-1} c_{i}\right] \\
\left.-k \mu_{4}^{2}\right)
\end{gathered}
$$

Where $\mu_{f-i}$ and $\sigma_{f-i}^{2}$ are given by (6). To verify real life signals like music and speech signals the proposed models can be used.

\section{CONCLUSiON}

In this paper the method of estimating the Signal Transition Activity in FIR Filters implemented by parallel Multiplier Accumulator based on Modified Booth Algorithm has been proposed. The DBT model is used to analyze the signal transition activity by means of the signal statistics. The mathematical formulation of the multiplexing mechanism is developed for the signal statistics. The mapping of FIR filter structure on a MAC based on modified booth algorithm increases the data throughput rate. The proposed multiplexing mechanism is used for the signal transition activity at the nodes. 


\section{REFERENCES}

[1] G. Lakshmi Narayanan and B. Venkataramani, "Optimization Techniques for FPGA-Based Wave Pipelined DSP Blocks", IEEE Trans. Very Large Scale Integr. (VLSI) Syst., 13. No 7. pp 783-792, July 2005.

[2] H. Lee, "A Power-Aware Scalable Pipelined Booth Multiplier", In Proc. IEEE Int. SOC Conf., 2004, pp. 123-126.

[3] P.E.Landman and J.M.Rabaey, "Architectural power analysis: The dual -bit-type Method", IEEE Trans. VLSI Syst., vol.3. pp 173-187, July 1995.

[4] D. Marculescu,R.Marculescu,and M.Pedram, "Information theoretic measures for power analysis", IEEE Trans. Computer-Aided Design.,vol. 15. pp 599-610, June 1996.

[5] S. Ramprasad, N.R.Shanbhag, and I.N. Hajj, "analytical estimation of signal transition from wordlevel staistics", IEEE Trans., Computer Aided Design,vol. 16, pp 718-733, July 1997.

[6] G. Lakshmi Narayanan and B. Venkataramani, "Optimization Techniques for FPGA-Based Wave Pipelined DSP Blocks", IEEE Trans. Very Large Scale Integr. (VLSI) Syst., 13. No 7. pp 783-792, July 2005.

[7] Young-Ho Seo and Dong-Wook Kim, "a New VLSI Architecture of parallel Multiplier-Accumulator Based on Radix-2 Modified Booth Algorithm”, IEEE Trans. Very Large Scale Integr. (VLSI) Syst., vol.18. No 2. pp 201-208, February 2010.

[8] A.Papoulis, "Probablity, Random variables, and Stochastic Processes", 3rd ed. New York:McGrawHill,1991. 